\title{
Software defect prediction: do different classifiers find the same defects?
}

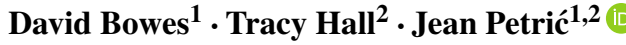

Published online: 7 February 2017

(C) The Author(s) 2017. This article is published with open access at Springerlink.com

\begin{abstract}
During the last 10 years, hundreds of different defect prediction models have been published. The performance of the classifiers used in these models is reported to be similar with models rarely performing above the predictive performance ceiling of about $80 \%$ recall. We investigate the individual defects that four classifiers predict and analyse the level of prediction uncertainty produced by these classifiers. We perform a sensitivity analysis to compare the performance of Random Forest, Naïve Bayes, RPart and SVM classifiers when predicting defects in NASA, open source and commercial datasets. The defect predictions that each classifier makes is captured in a confusion matrix and the prediction uncertainty of each classifier is compared. Despite similar predictive performance values for these four classifiers, each detects different sets of defects. Some classifiers are more consistent in predicting defects than others. Our results confirm that a unique subset of defects can be detected by specific classifiers. However, while some classifiers are consistent in the predictions they make, other classifiers vary in their predictions. Given our results, we conclude that classifier ensembles with decision-making strategies not based on majority voting are likely to perform best in defect prediction.
\end{abstract}

Keywords Software defect prediction · Prediction modelling · Machine learning

Jean Petrić

j.petric@herts.ac.uk

David Bowes

d.h.bowes@herts.ac.uk

Tracy Hall

tracy.hall@brunel.ac.uk

1 Science and Technology Research Institute, University of Hertfordshire, Hatfield, Hertfordshire, AL10 9AB, UK

2 Department of Computer Science, Brunel University London, Uxbridge, Middlesex, Uxbridge UB8 3PH, UK 


\section{Introduction}

Defect prediction models can be used to direct test effort to defect-prone code. ${ }^{1}$ Latent defects can then be detected in code before the system is delivered to users. Once found, these defects can be fixed pre-delivery, at a fraction of post-delivery fix costs. Each year, defects in code cost industry billions of dollars to find and fix. Models which efficiently predict where defects are in code have the potential to save companies large amounts of money. Because the costs are so huge, even small improvements in our ability to find and fix defects can make a significant difference to overall costs. This potential to reduce costs has led to a proliferation of models which predict where defects are likely to be located in code. Hall et al. (2012) provide an overview of several hundred defect prediction models published in 208 studies.

Traditional defect prediction models comprise of four main elements. First, the model uses independent variables (or predictors) such as static code features, change data or previous defect information on which to base its predictions about the potential defect proneness of a unit of code. Second, the model is based on a specific modelling technique. Modelling techniques are mainly either machine learning (classification) or regression methods ${ }^{2}$ (Wahono 2015). Third, dependent variables (or prediction outcomes) are produced by the model which are usually either categorical predictions (i.e. a code unit is predicted as either defect prone or not defect prone) or continuous predictions (i.e. the number of defects are predicted in a code unit). Fourth, a scheme is designed to measure the predictive performance of a model. Measures based on the confusion matrix are often used for categorical predictions and measures related to predictive error are often used for continuous predictions.

The aim of this paper is to identify classification techniques which perform well in software defect prediction. We focus on within-project prediction as this is a very common form of defect prediction. Many eminent researchers before us have also aimed to do this (e.g. Briand et al. (2002) and Lessmann et al. (2008)). Those before us have differentiated predictive performance using some form of measurement scheme. Such schemes typically calculate performance values (e.g. precision and recall ; see Table 3) to calculate an overall number representing how well models correctly predict truly defective and truly non-defective codes taking into account the level of incorrect predictions made. We go beyond this by looking underneath the numbers and at the individual defects that specific classifiers detect and do not detect. We show that, despite the overall figures suggesting similar predictive performances, there is a marked difference between four classifiers in terms of the specific defects each detects and does not detect. We also investigate the effect of prediction 'flipping' among these four classifiers. Although different classifiers can detect different subsets of defects, we show that the consistency of predictions vary greatly among the classifiers. In terms of prediction consistency, some classifiers tend to be more stable when predicting a specific software unit as defective or non-defective, hence 'flipping' less between experiment runs.

\footnotetext{
${ }^{1}$ Defects can occur in many software artefacts, but here, we focus only on defects found in code.

${ }^{2}$ In this paper, we concentrate on classification models only. Hall et al. (2012) show that about $50 \%$ of prediction models are based on classification techniques. We do this because a totally different set of analysis techniques is needed to investigate the outcomes of regression techniques. Such an analysis is beyond the scope of this paper.
} 
Identifying the defects that different classifiers detect is important as it is well known (Fenton and Neil 1999) that some defects matter more than others. Identifying defects with critical effects on a system is more important than identifying trivial defects. Our results offer future researchers an opportunity to identify classifiers with capabilities to identify sets of defects that matter most. Panichella et al. (2014) previously investigated the usefulness of a combined approach to identifying different sets of individual defects that different classifiers can detect. We build on (Panichella et al. 2014) by further investigating whether different classifiers are equally consistent in their predictive performances. Our results confirm that the way forward in building high-performance prediction models in the future is by using ensembles (Kim et al. 2011). Our results also show that researchers should repeat their experiments a sufficient number of times to avoid the 'flipping' effect that may skew prediction performance.

We compare the predictive performance of four classifiers: Naïve Bayes, Random Forest, RPart and Support Vector Machines (SVM). These classifiers were chosen as they are widely used by the machine-learning community and have been commonly used in previous studies. These classifiers offer an opportunity to compare the performance of our classification models against those in previous studies. These classifiers also use distinct predictive techniques, and so, it is reasonable to investigate whether different defects are detected by each and whether the prediction consistency is distinct among the classifiers.

We apply these four classifiers to twelve NASA datasets, ${ }^{3}$ three open source datasets, ${ }^{4}$ and three commercial datasets from our industrial partner (see Table 1). NASA datasets provide a standard set of independent variables (static code metrics) and dependent variables (defect data labels). NASA data modules are at a function level of granularity. Additionally, we analyse the open source systems: Ant, Ivy, and Tomcat from the PROMISE repository (Jureczko and Madeyski 2010). Each of these datasets is at the class level of granularity. We also use three commercial telecommunication datasets which are at a method level. Therefore, our analysis includes datasets with different metrics granularity and from different software domains.

This paper extends our earlier work (Bowes et al. 2015). We build on our previous findings by adding more datasets into our experimental set-up and validating the conclusions previously made. To the NASA datasets used in Bowes et al. (2015), we add six new datasets (three open source, and three industrial datasets). Introducing more datasets to our analysis increases the diversity of code and defects in those systems. Confirming our previous findings with increased and diverse datasets provides evidence that our results may be generalisable.

The following section is an overview of defect prediction. Section 3 details our methodology. Section 4 presents results which are discussed in Section 5. We identify threats to validity in Section 6 and conclude in Section 7.

\section{Background}

Many studies of software defect prediction have been performed over the years. In 1999, Fenton and Neil critically reviewed a cross section of such studies (Fenton and Neil 1999). Catal and Diri (2009) mapping study identified 74 studies, and in our more recent study

\footnotetext{
${ }^{3} \mathrm{http}: / /$ promisedata.googlecode.com/svn/trunk/defect/

${ }^{4} \mathrm{http} / / /$ openscience.us/repo/defect/ck/
} 
Table 1 Summary statistics for datasets before and after cleaning

\begin{tabular}{|c|c|c|c|c|c|c|c|c|}
\hline Project & Dataset & Language & $\begin{array}{l}\text { Total } \\
\text { KLOC }\end{array}$ & $\begin{array}{l}\text { No. of } \\
\text { modules } \\
\text { (pre- } \\
\text { cleaning) }\end{array}$ & $\begin{array}{l}\text { No. of } \\
\text { modules } \\
\text { (post- } \\
\text { cleaning) }\end{array}$ & $\begin{array}{l}\% \text { loss due } \\
\text { to cleaning }\end{array}$ & $\begin{array}{l}\text { \%faulty } \\
\text { modules } \\
\text { (pre- } \\
\text { cleaning) }\end{array}$ & $\begin{array}{l}\text { \%faulty } \\
\text { modules } \\
\text { (post- } \\
\text { cleaning) }\end{array}$ \\
\hline $\begin{array}{l}\text { Spacecraft } \\
\text { instrumentation }\end{array}$ & CM1 & $\mathrm{C}$ & 20 & 505 & 505 & 0.0 & 9.5 & 9.5 \\
\hline Ground data & $\mathrm{KC} 1$ & $\mathrm{C}++$ & 43 & 2109 & 2096 & 0.6 & 15.4 & 15.5 \\
\hline Storage & $\mathrm{KC} 3$ & Java & 18 & 458 & 458 & 0.0 & 9.4 & 9.4 \\
\hline Management & $\mathrm{KC} 4$ & Perl & 25 & 125 & 125 & 0.0 & 48.8 & 48.8 \\
\hline Combustion & $\mathrm{MC} 1$ & $\mathrm{C} \& \mathrm{C}++$ & 63 & 9466 & 9277 & 2.0 & 0.7 & 0.7 \\
\hline Experiment & $\mathrm{MC} 2$ & $\mathrm{C}$ & 6 & 161 & 161 & 1.2 & 32.3 & 32.3 \\
\hline $\begin{array}{l}\text { Zero gravity } \\
\text { experiment }\end{array}$ & MW1 & $\mathrm{C}$ & 8 & 403 & 403 & 0.0 & 7.7 & 7.7 \\
\hline Flight software & PC1 & $\mathrm{C}$ & 40 & 1107 & 1107 & 0.0 & 6.9 & 6.9 \\
\hline for Earth & $\mathrm{PC} 2$ & $\mathrm{C}$ & 26 & 5589 & 5460 & 2.3 & 0.4 & 0.4 \\
\hline orbiting & PC3 & $\mathrm{C}$ & 40 & 1563 & 1563 & 0.0 & 10.2 & 0.0 \\
\hline \multirow[t]{2}{*}{ satellites } & $\mathrm{PC} 4$ & $\mathrm{C}$ & 36 & 1458 & 1399 & 4.0 & 12.2 & 12.7 \\
\hline & PC5 & $\mathrm{C}++$ & 164 & 17186 & 17001 & 1.1 & 3.0 & 3.0 \\
\hline $\begin{array}{l}\text { Real-time } \\
\text { predictive } \\
\text { ground } \\
\text { system }\end{array}$ & JM1 & $\mathrm{C}$ & 315 & 10878 & 7722 & 29.0 & 19.0 & 21.0 \\
\hline Telecommunication & $\mathrm{PA}$ & Java & 21 & 4996 & 4996 & 0.0 & 11.7 & 11.7 \\
\hline \multirow[t]{2}{*}{ Software } & $\mathrm{KN}$ & Java & 18 & 4314 & 4314 & 0.0 & 7.5 & 7.5 \\
\hline & HA & Java & 43 & 9062 & 9062 & 0.0 & 1.3 & 1.3 \\
\hline Java build tool & Ant & Java & 209 & 745 & 742 & 0.0 & 22.3 & 22.4 \\
\hline $\begin{array}{l}\text { Dependency } \\
\text { manager }\end{array}$ & Ivy & Java & 88 & 352 & 352 & 0.0 & 11.4 & 11.4 \\
\hline Web server & Tomcat & Java & 301 & 858 & 852 & 0.0 & 9.0 & 9.0 \\
\hline
\end{tabular}

(Hall et al. 2012), we systematically reviewed 208 primary studies and showed that predictive performance varied significantly between studies. The impact that many aspects of defect models have on predictive performance have been extensively studied.

The impact that various independent variables have on predictive performance has been the subject of a great deal of research effort. The independent variables used in previous studies mainly fall into the categories of product (e.g. static code data) metrics and process (e.g. previous change and defect data) as well as metrics relating to developers. Complexity metrics are commonly used (Zhou et al. 2010), but LOC is probably the most commonly used static code metric. The effectiveness of LOC as a predictive independent variable remains unclear. Zhang (2009) reports LOC to be a useful early general indicator of defect proneness. Other studies report LOC data to have poor predictive power and is out-performed by other metrics (e.g. Bell et al. (2006)). Malhotra (2015) suggests that object-oriented metrics such as coupling between objects and response for a class are useful for defect prediction. 
Several previous studies report that process data, in the form of previous history data, performs well (e.g. D’Ambros et al. (2009), Shin et al. (2009), Nagappan et al. (2010), and Madeyski and Jureczko (2015)). D'Ambros et al. (2009) specifically report that previous bug reports are the best predictors. More sophisticated process measures have also been reported to perform well (e.g. Nagappan et al. (2010)). In particular, Nagappan et al. (2010) use 'change burst' metrics with which they demonstrate good predictive performance. The few studies using developer information in models report conflicting results. Ostrand et al. (2010) report that the addition of developer information does not improve predictive performance much. Bird et al. (2009b) report better performances when developer information is used as an element within a socio-technical network of variables. Madeyski and Jureczko (2015) show that some process metrics are particularly useful for predictive modelling. For example, the number of developers changing a file can significantly improve defect prediction (Madeyski and Jureczko 2015). Many other independent variables have also been used in studies, for example Mizuno et al. (2007) and Mizuno and Kikuno (2007) use the text of the source code itself as the independent variable with promising results.

Lots of different datasets have been used in studies. However, our previous review of 208 studies (Hall et al. 2012) suggests that almost $70 \%$ of studies have used either the Eclipse dataset. ${ }^{5,6}$ Wahono (2015) and Kamei and Shihab (2016) suggest that the NASA datasets remain the most popular for defect prediction, and also report that the PROMISE repository is used increasingly. Ease of availability mean that these datasets remain popular despite reported issues of data quality. Bird et al. (2009a) identifies many missing defects in the Eclipse data. While Gray et al. (2012), Boetticher (2006), and Shepperd et al. (2013), and Petrić et al. (2016b) raise concerns over the quality of NASA datasets in the original PROMISE repository. ${ }^{7}$ Datasets can have a significant effect on predictive performance. Some datasets seem to be much more difficult than others to learn from. The PC2 NASA dataset seems to be particularly difficult to learn from. Kutlubay et al. (2007) and Menzies et al. (2007) both note this difficulty and report poor predictive results using these datasets. As a result, the PC2 dataset is more seldom used than other NASA datasets. Another example of datasets that are difficult to predict from are those used by Arisholm and Briand (2007) and Arisholm et al. (2010). Very low precision is reported in both of these Arisholm et al. studies (as shown in Hall et al. (2012)). Arisholm and Briand (2007) and Arisholm et al. (2010) report many good modelling practices and in some ways are exemplary studies. But these studies demonstrate how the data used can impact significantly on the performance of a model.

It is important that defect prediction studies consider the quality of data on which models are built. Datasets are often noisy. They often contain outliers and missing values that can skew results. Confidence in the predictions made by a model can be impacted by the quality of the data used while building the model. For example, Gray et al. (2012) show that defect predictions can be compromised where there is a lack of data cleaning with Jiang et al. (2009) acknowledging the importance of data quality. Unfortunately, Liebchen and Shepperd (2008) report that many studies do not seem to consider the quality of the data they use, but that small problems with data quality can have a significant impact on results.

\footnotetext{
${ }^{5}$ http://www.st.cs.uni-saarland.de/softevo/bug-data/eclipse/

${ }^{6}$ https://code.google.com/p/promisedata/(Menzies et al. 2012)

${ }^{7}$ http://promisedata.org
} 
The features of the data also need to be considered when building a defect prediction model. In particular, repeated attributes and related attributes have been shown to bias the predictions of models. The use of feature selection on sets of independent variables seems to improve the performance of models (e.g. Shivaji et al. (2009), Khoshgoftaar et al. (2010), Bird et al. (2009b), and Menzies et al. (2007)).

Data balance is also an important factor in defect prediction and has been considered by previous studies. This is important as substantially imbalanced datasets are commonly used in defect prediction studies (i.e. there are usually many more non-defective units than defective units) (Bowes et al. 2013; Myrtveit et al. 2005). An extreme example of this is seen in NASA dataset PC2, which has only $0.4 \%$ of datapoints belonging to the defective class (23 out of 5589 datapoints). Imbalanced data can strongly influence both the training of a model, and the suitability of performance metrics. The influence data imbalance has on predictive performance varies from one classifier to another. For example, C4.5 decision trees have been reported to struggle with imbalanced data (Chawla et al. 2004; Arisholm and Briand 2007; Arisholm et al. 2010), whereas fuzzy-based classifiers have been reported to perform robustly regardless of class distribution (Visa and Ralescu 2004). Data balancing has shown positive effects when used with Random Forest (Chen et al. 2014); however, there is a risk of over-fitting (Gray et al. 2012). On the other hand, data balancing has demonstrated no significant effect on performance when used with some other techniques (e.g. Naïve Bayes (Rodriguez et al. 2014)). Studies specifically investigating the impact of defect data balance and proposing techniques to deal with it include, for example, Khoshgoftaar et al. (2010), Shivaji et al. (2009), and Seiffert et al. (2009). Gao et al. (2015) investigate the combination of feature selection and data balancing techniques. Particularly, Gao et al. (2015) experiment with changing the order of the two techniques, using feature selection followed by data balancing (separately using sampled and unsampled data instances), and vice versa. They show that sampling performed prior to feature selection by keeping the unsampled data can boost prediction performance more than the other two approaches (Gao et al. 2015).

Classifiers are mathematical techniques for building models which can then predict dependent variables (defects). Defect prediction has frequently used trainable classifiers (Wahono 2015). Trainable classifiers build models using training data which has items composed of both independent and dependant variables. There are many classification techniques that have been used in previous defect prediction studies. Witten (2005) explain classification techniques in detail and Lessmann et al. (2008) summarise the use of 22 such classifiers for defect prediction. Ensembles of classifiers are also used in prediction (Minku and Yao 2012; Sun et al. 2012; Laradji et al. 2015; Petrić et al. 2016a). Ensembles are collections of individual classifiers trained on the same data and combined to perform a prediction task. An overall prediction decision is made by the ensemble based on the predictions of the individual models. Majority voting is a decision-making strategy commonly used by ensembles. Although not yet widely used in defect prediction, ensembles have been shown to significantly improve predictive performance. For example, Misirlı et al. (2011) combine the use of Artificial Neural Networks, Naïve Bayes and Voting Feature Intervals and report improved predictive performance over the individual models. Ensembles have been more commonly used to predict software effort estimation (e.g. Minku and Yao (2013)) where their performance has been reported as sensitive to the characteristics of datasets (Chen and Yao 2009; Shepperd and Kadoda 2001).

Many defect prediction studies individually report the comparative performance of the classification techniques they have used. Mizuno and Kikuno (2007) report that, of the techniques they studied, Orthogonal Sparse Bigrams Markov models (OSB) are best suited to defect prediction. Bibi et al. (2006) report that Regression via Classification works well. 
Khoshgoftaar et al. (2002) report that modules whose defect proneness is predicted as uncertain, can be effectively classified using the TreeDisc technique. Our own analysis of the results from 19 studies (Hall et al. 2012) suggests that Naïve Bayes and Logistic regression techniques work best. However, overall, there is no clear consensus on which techniques perform best. Several influential studies have performed large-scale experiments using a wide range of classifiers to establish which classifiers dominate. In Arisholm et al. (2010) systematic study of the impact that classifiers, metrics and performance measures have on predictive performance, eight classifiers were evaluated. Arisholm et al. (2010) report that the classifier technique had limited impact on predictive performance. Lessmann et al. (2008) large-scale comparison of predictive performance across 22 classifiers over 10 NASA datasets showed no significant performance differences among the top 17 classifiers.

In general, defect prediction studies do not consider individual defects that different classifiers predict or do not predict. Panichella et al. (2014) is an exception to this reporting a comprehensive empirical investigation into whether different classifiers find different defects. Although predictive performances among the classifiers in their study were similar, they showed that different classifiers detect different defects. Panichella et al. proposed CODEP which uses an ensemble technique (i.e. stacking Wolpert (1992)) to combine multiple learners in order to achieve better predictive performances. The CODEP model showed superior results when compared to single models. However, Panichella et al. conducted a cross-project defect prediction study which differs from our study. Cross-project defect prediction has an experimental set-up based on training models on multiple projects and then tested on one project (explanatory studies on cross-project defect prediction were done by Turhan et al. (2009) and Zimmermann et al. (2009)). Consequently, in cross-project defect prediction studies, the multiple execution of experiments is not required. Contrary, in within-project defect prediction studies, experiments are frequently done using crossvalidation techniques. To get more stabilised and generalised results, experiments based on cross validation are repeated multiple times. As a drawback of executing experiments multiple times, the prediction consistency may not be stable resulting in classifiers 'flipping' between experimental runs. Therefore, in a within-project analysis, prediction consistency should also be taken into account.

Our paper further builds on Panichella et al. in a number of other ways. Panichella et al. conducted an analysis only at a class level while our study is additionally extended to a module level (i.e. the smallest unit of functionality, usually a function, procedure or method). Panichella et al. also consider regression analysis where probabilities of a module being defective are calculated. Our study deals with classification where a module is labelled either as defective or non-defective. Therefore, the learning algorithms used in each study differ. We also show full performance figures by presenting the numbers of true positives, false positives, true negative and false negatives for each classifier.

Predictive performance in all previous studies is presented in terms of a range of performance measures (see the following sub-sections for more details of such measures). The vast majority of predictive performances were reported to be within the current performance ceiling of $80 \%$ recall identified by Menzies et al. (2008). However, focusing only on performance figures, without examining the individual defects that individual classifiers detect, is limiting. Such an approach makes it difficult to establish whether specific defects are consistently missed by all classifiers, or whether different classifiers detect different subsets of defects. Establishing the set of defects each classifier detects, rather than just looking at the overall performance figure, allows the identification classifier ensembles most likely to detect the largest range of defects. 
Table 2 Confusion matrix

The confusion matrix is in many ways analogous to residuals for regression models. It forms the fundamental basis from which almost all other performance statistics are derived.

\begin{tabular}{lll}
\hline & Predicted defective & Predicted defect free \\
\hline $\begin{array}{l}\text { Observed } \\
\text { defective }\end{array}$ & True positive & False negative \\
Observed & (TP) & (FN) \\
defect free & False positive & True negative \\
\hline
\end{tabular}

Studies present the predictive performance of their models using some form of measurement scheme. Measuring model performance is complex and there are many ways in which the performance of a prediction model can be measured. For example, Menzies et al. (2007) use pd and pf to highlight standard predictive performance, while Mende and Koschke (2010) use Popt to assess effort-awareness. The measurement of predictive performance is often based on a confusion matrix (shown in Table 2). This matrix reports how a model classified the different defect categories compared to their actual classification (predicted versus observed). Composite performance measures can be calculated by combining values from the confusion matrix (see Table 3).

There is no one best way to measure the performance of a model. This depends on the distribution of the training data, how the model has been built and how the model will be used. For example, the importance of measuring misclassification will vary depending on the application. Zhou et al. (2010) report that the use of some measures, in the context of a particular model, can present a misleading picture of predictive performance and undermine the reliability of predictions. Arisholm et al. (2010) also discuss how model performance varies depending on how it is measured. The different performance measurement schemes used mean that directly comparing the performance reported by individual studies is difficult and potentially misleading. Comparisons cannot compare like with like as there is no adequate point of comparison. To allow such comparisons, we previously developed a tool to transform a variety of reported predictive performance measures back to a confusion matrix (Bowes et al. 2013).

Table 3 Composite performance measures

\begin{tabular}{|c|c|c|}
\hline Construct & Defined as & Description \\
\hline $\begin{array}{l}\text { Recall pd (probability of } \\
\text { detection) sensitivity true } \\
\text { positive rate }\end{array}$ & $T P /(T P+F N)$ & $\begin{array}{l}\text { Proportion of defective units cor- } \\
\text { rectly classified }\end{array}$ \\
\hline Precision & $T P /(T P+F P)$ & $\begin{array}{l}\text { Proportion of units correctly pre- } \\
\text { dicted as defective }\end{array}$ \\
\hline F-measure & $\frac{2 \cdot \text { Recall } \cdot \text { Precision }}{\text { Recall }+ \text { Precision }}$ & $\begin{array}{l}\text { Most commonly defined as the har- } \\
\text { monic mean of precision and recall }\end{array}$ \\
\hline $\begin{array}{l}\text { Matthews correlation } \\
\text { coefficient }\end{array}$ & $\frac{T P \times T N-F P \times F N}{\sqrt{(T P+F P)(T P+F N)(T N+F P)(T N+F N)}}$ & $\begin{array}{l}\text { Combines all quadrants of the binary } \\
\text { confusion matrix to produce a value } \\
\text { in the range }-1 \text { to }+1 \text { with } 0 \text { indicat- } \\
\text { ing random correlation between the } \\
\text { prediction and the recorded results. } \\
\text { MCC can be tested for statistical sig- } \\
\text { nificance, with } \chi^{2}=N \cdot M C C^{2} \\
\text { where } N \text { is the total number of } \\
\text { instances. }\end{array}$ \\
\hline
\end{tabular}




\section{Methodology}

\subsection{Classifiers}

We have chosen four different classifiers for this study: Naïve Bayes, RPart, SVM and Random Forest. These four classifiers were chosen because they build models based on different mathematical properties. Naïve Bayes produces models based on the combined probabilities of a dependent variable being associated with the different categories of the dependent variables. Naïve Bayes requires that both the dependent and independent variables are categorical. RPart is an implementation of a technique for building Classification and Regression Trees (CaRT). RPart builds a decision tree based on the information entropy (uniformity) of the subsets of training data which can be achieved by splitting the data using different independent variables. SVMs build models by producing a hyper-plane which can separate the training data into two classes. The items (vectors) which are closest to the hyper-plane are used to modify the model with the aim of producing a hyper-plane which has the greatest average distance from the supporting vectors. Random Forest is an ensemble technique. It is built by producing many CaRTs, each with samples of the training data having a subset of features. Bagging is also used to improve the stability of the individual trees by creating training sets produced by sampling the original training data with replacement. The final decision of the ensemble is determined by combining the decisions of each tree and computing the modal value.

The different methods of building a model by each classifier may lead to differences in the items predicted as defective. Naïve Bayes is purely probabilistic and each independent variable contributes to a decision. RPart may use only a subset of independent variables to produce the final tree. The decisions at each node of the tree are linear in nature and collectively put boundaries around different groups of items in the original training data. RPart is different to Naïve Bayes in that the thresholds used to separate the groups are different at each node compared to Naïve Bayes which decides the threshold to split continuous variables before the probabilities are determined. SVMs use mathematical formulae to build nonlinear models to separate the different classes. The model is therefore not derived from decisions based on individual independent variables, but on the ability to find a formula which separates the data with the least amount of false negatives and false positives.

Classifier tuning is an important part of building good models. As described above, Naïve Bayes requires all variables to be categorical. Choosing arbitrary threshold values to split a continuous variable into different groups may not produce good models. Choosing good thresholds may require many models to be built on the training data using different threshold values and determining which produces the best results. Similarly for RPart, the number of items in the leaf nodes of a tree should not be so small that a branch is built for every item. Finding the minimum number of items required before branching is an important process in building good models which do not overfit on the training data and then do not perform as well on the test data. Random Forest can be tuned to determine the most appropriate number of trees to use in the forest. Finally SVMs are known to perform poorly if they are not tuned (Soares et al. 2004). SVMs can use different kernel functions to produce the complex hyperplanes needed to separate the data. The radial-based kernel function has two parameters: $C$ and $\gamma$, which need to be tuned in order to produce good models.

In practice, not all classifiers perform significantly better when tuned. Both Naïve Bayes and RPart can be tuned, but the default parameters and splitting algorithms are known to work well. Random Forest and particularly SVMs do require tuning. For Random Forest we tuned the number of trees from 50 to 200 in steps of 50. For SVM using a radial base 
function, we tuned $\gamma$ from 0.25 to 4 and $C$ from 2 to 32. In our experiment, tuning was carried out by splitting the training data into 10 folds, 9 folds were combined together to build models with the parameters and the 10th fold was used to measure the performance of the model. This was repeated with each fold being held out in turn. The parameters which produced the best average performance we used to build the final model on the entire training data.

\subsection{Datasets}

We used the NASA datasets first published on the now defunct MDP website. ${ }^{8}$ This repository consists of 13 datasets from a range of NASA projects. In this study, we use 12 of the 13 NASA datasets. JM1 was not used because during cleaning, 29\% of data was removed suggesting that the quality of the data may have been poor. We extended our previous analysis (Bowes et al. 2015) by using 6 additional datasets, 3 open source and 3 commercial. All 3 open source datasets are at class level, and originate from the PROMISE repository. The commercial datasets are all in the telecommunication domain and are at method level. A summary of each dataset can be found in Table 1. Our choice of datasets is based on several factors. First, the NASA and PROMISE datasets are frequently used in defect prediction. Second, three open source datasets in our analysis (Ant, Ivy, and Tomcat) are very different in nature, and they could have a variety of different defects. Commercial datasets also add to the variety of very different datasets. We use these factors to enhance the possibility of generalising our results, which is one of the major contributions of this paper.

The data quality of the original NASA MDP datasets can be improved (Boetticher 2006; Gray et al. 2012; Shepperd et al. 2013). Gray et al. (2012), Gray (2013), and Shepperd et al. (2013) describe techniques for cleaning the data. Shepperd has provided a 'cleaned' version of the MDP datasets, ${ }^{9}$ However, full traceability back to the original items is not provided. Consequently we did not use Shepperd's cleaned NASA datasets. Instead we cleaned the NASA datasets ourselves. We carried out the following data cleaning stages described by Gray et al. (2012): Each independent variable was tested to see if all values were the same; if they were, this variable was removed because they contained no information which allows us to discriminate defective items from non-defective items. The correlation for all combinations of two independent variables was found; if the correlation was 1 , the second variable was removed. Where the dataset contained the variable 'DECISION DENSITY', any item with a value of 'na' was converted to 0 . The 'DECISION_DENSITY' was also set to 0 if 'CONDITION_COUNT' $=0$ and 'DECISION_COUNT' $=0$. Items were removed if:

\section{HALSTEAD_LENGTH!= NUM_OPERANDS+NUM_OPERATORS \\ 2. CYCLOMATIC_COMPLEXITY $>1+$ NUM_OPERATORS \\ 3. CALL_PAIRS $>$ NUM_OPERATORS}

Our method for cleaning the NASA data also differs from Shepperd et al. (2013) because we do not remove items where the executable lines of code is zero. We did not do this because we have not been able to determine how the NASA metrics were computed and it is possible to have zero executable lines in Java interfaces. We performed the same cleaning to our commercial datasets. We performed cleaning of the open source datasets for which

\footnotetext{
${ }^{8}$ http://mdp.ivv.nasa.gov - unfortunately now not accessible

${ }^{9}$ http://nasa-softwaredefectdatasets.wikispaces.com
} 
we defined a similar set of rules as described above, for data at a class level. Particularly, we removed items if:

\section{AVERAGE_CYCLOMATIC_COMPLEXITY> MAXIMAL_CYCLOMATIC_COMPLEXITY \\ 2. NUMBER_OF_COMMENTS $>$ LINES_OF_CODE \\ 3. PUBLIC_METODS_COUNT $>$ CLASS_METHODS_COUNT}

Since all the NASA and open source datasets are publicly available, the aforementioned cleaning steps can be applied to them. We do not provide pre-cleaned data as we believe it is vital that researchers do their own cleaning. Problems with poor-quality data being used have prolificated because researchers have taken pre-cleaned data without questioning their quality (for example NASA datasets (Gray et al. 2011; Shepperd et al. 2013)). Cleaning the data we use is straightforward to future researchers as the cleaning steps are easy to understand and implement.

\subsection{Experimental Set-Up}

The following experiment was repeated 100 times. Experiments are more commonly repeated 10 times. We chose 100 repeats because Mende (2011) reports that using 10 experiment repeats results in an unreliable final performance figure. Each dataset was split into 10 stratified folds. Each fold was held out in turn to form a test set and the other folds were combined and randomised (to reduce ordering effects) to produce the training set. Such stratified cross validation ensures that there are instances of the defective class in each test set, so reduces the likelihood of classification uncertainty. Re-balancing of the training set is sometimes carried out to provide the classifier with a more representative sample of the infrequent defective instances. Data balancing may have very different effects on an experiment depending on the classifier used (as explained in Section 2). To reduce the confounding factors of data balance, we do not apply this technique in our experiment. Specifically, it would be difficult to control the impact of data balance on performance across the range of classifiers we used. Also, our experiment is focused on the dispersion of individual predictions based on real data across classifiers, rather than investigating whether and how re-balancing affects defect prediction results. Furthermore, data balancing is infrequently used in defect prediction studies. For each training/testing pair, four different classifiers were trained using the same training set. Where appropriate, a grid search was performed to identify optimal meta-parameters for each classifier on the training set. The model built by each classifier was used to classify the test set.

To collect the data showing individual predictions made by individual classifiers, the RowID, DataSet, runid, foldid and classified label (defective or not defective) was recorded for each item in the test set for each classifier and for each cross-validation run.

We calculate predictive performance values using two different measures: f-measure and MCC (see Table 3). F-measure was selected because it is very commonly used by published studies and allows us to easily compare the predictive performance of our models against previous models. Additionally, f-measure gives the harmonic mean of both measures, precision and recall. It has a range of 0 to 1 . MCC was selected because it is relatively easy to understand with a range from -1 to +1 . MCC has the added benefit that it encompasses all four components of the confusion matrix whereas f-measure ignores the proportion of true negatives. Furthermore, Matthews' Correlation Coefficient (MCC) has been demonstrated to be a reliable measure of predictive model performance (Shepperd et al. 2014). The results for each combination of classifier and dataset were further analysed by calculating for each 


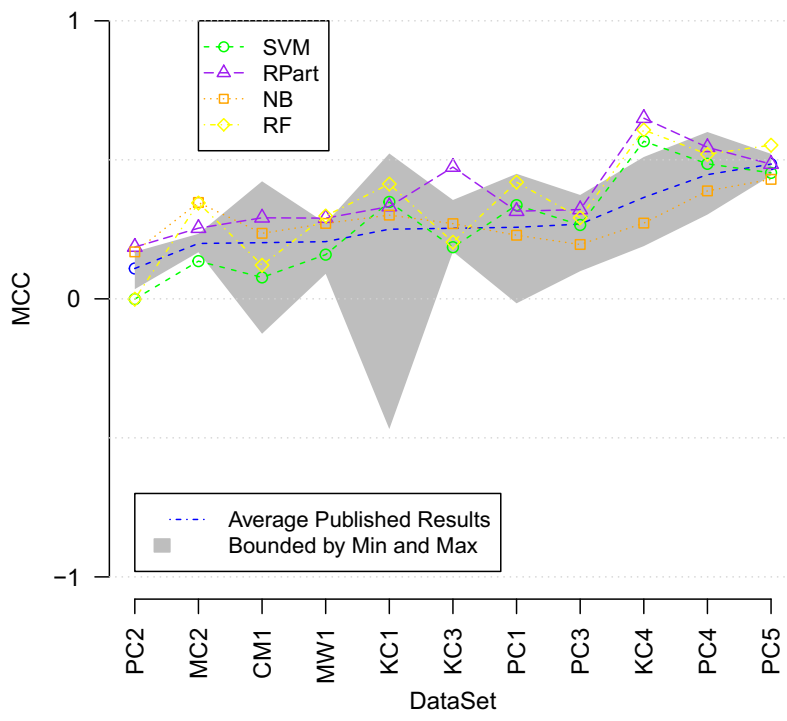

Fig. 1 Our results compared to results published by other studies

item the frequency of being classified as defective. The results were then categorised by the original label for each item so that we can see the difference between how the models had classified the defective and non-defective items.

\section{Results}

We aim to investigate variation in the individual defects and prediction consistency produced by the four classifiers. To ensure the defects that we analyse are reliable, we first checked that our models were performing satisfactorily. To do this, we built prediction models using the NASA datasets. Figure 1 compares the MCC performance of our models against 600 defect prediction performances reported in published studies using these NASA datasets Hall et al. (2012). ${ }^{10}$ We re-engineered MCC from the performance figures reported in these previous studies using DConfusion. This is a tool we developed for transforming a variety of reported predictive performance measures back to a confusion matrix. DConfusion is described in (Bowes et al. 2013). Figure 1 shows that the performances of our four classifiers are generally in keeping with those reported by others. Figure 1 confirms that some datasets are notoriously difficult to predict. For example, few performances for PC2 are better than random. Whereas, very good predictive performances are generally reported for PC5 and KC4. The RPart and Naïve Bayes classifiers did not perform as well on the NASA datasets as on our commercial datasets (as shown in Table 4). However, all our commercial datasets are highly imbalanced, where learning from a small set of defective items becomes more difficult, so this imbalance may explain the difference in the way these two classifiers perform. Similarly the SVM classifier performs better on the open source datasets than it does on the NASA datasets. The SVM classifier seems to perform particularly poorly when

\footnotetext{
${ }^{10}$ Dataset $\mathrm{MC} 1$ is not included in the figure because none of the studies we had identified previously used this dataset.
} 
Table 4 MCC performance for all datasets by classifier

\begin{tabular}{|c|c|c|c|c|c|c|c|c|}
\hline \multirow[b]{2}{*}{ Classifier } & \multicolumn{2}{|c|}{ NASA datasets } & \multicolumn{2}{|c|}{ OSS datasets } & \multicolumn{2}{|c|}{ Commercial datasets } & \multicolumn{2}{|c|}{ All datasets } \\
\hline & Average & StDev & Average & StDev & Average & StDev & Average & StDev \\
\hline SVM & 0.291 & 0.188 & 0.129 & 0.134 & 0.314 & 0.140 & 0.245 & 0.154 \\
\hline RPart & 0.331 & 0.162 & 0.323 & 0.077 & 0.166 & 0.148 & 0.273 & 0.129 \\
\hline NB & 0.269 & 0.083 & 0.322 & 0.089 & 0.101 & 0.040 & 0.231 & 0.071 \\
\hline $\mathrm{RF}$ & 0.356 & 0.184 & 0.365 & 0.095 & 0.366 & 0.142 & 0.362 & 0.140 \\
\hline
\end{tabular}

used on extremely imbalanced datasets (especially the case when datasets have less than $10 \%$ faulty items).

We investigated classifier performance variation across all the datasets. Table 4 shows little overall difference in average MCC performance across the four classifiers, except Random Forest, which usually performs best (Lessmann et al. 2008). By using Friedman's non-parametric test at the significance level 0.05 , we formally confirmed no statistically significant difference in the MCC performance values across all datasets when applied amongst SVM, Naïve Bayes, and RPart classifiers (p-value: 0.939). Similarly, by using the same procedure, we established that there is a statistically significant difference in terms of MCC performance when Random Forest is added to the statistical test ( $p$ value: 0.021). However, these overall performance figures mask a range of different performances by classifiers when used on individual datasets. For example, Table 5 shows Naïve Bayes performing relatively well when used on the Ivy and KC4 datasets, however, much worse on the KN dataset. On the other hand, SVM achieves the highest MCC performance across all classifiers on the $\mathrm{KN}$ dataset, but poor performance values on the Ivy dataset. ${ }^{11}$ By repeating Friedman's non-parametric statistical test on the three datasets reported in Table 5, across all runs, we confirmed a statistically significant difference amongst the four classifiers, with the $p$ value less than 0.0001 in the cases where Random Forest was included or excluded from the test.

Having established that our models were performing acceptably (comparable to the 600 models reported in Hall et al. (2012) and depicted in Fig. 1), we next wanted to identify the particular defects that each of our four classifiers predicts so that we could identify variations in the defects predicted by each. We needed to be able to label each module as either containing a predicted defect (or not) by each classifier. As we used 100 repeated 10fold cross-validation experiments, we needed to decide on a prediction threshold at which we would label a module as either predicted defective (or not) by each classifier, i.e. how many of these 100 runs must have predicted that a module was defective before we labelled it as such. We analysed the labels that each classifier assigned to each module for each of the 100 runs. There was a surprising amount of prediction 'flipping' between runs. On some runs, a module was labelled as defective and other runs not. There was variation in the level of prediction flipping amongst the classifiers. Table 7 shows the overall label 'flipping' between the classifiers.

Table 6 divides predictions between the actual defective and non-defective labels (i.e. the known labels for each module) for each of our dataset category, namely NASA, commercial (Comm), and open source dataset (OSS), respectively. For each of these two categories, Table 6 shows three levels of label flipping: never, $5 \%$ and $10 \%$. For example, a value of

\footnotetext{
${ }^{11}$ Performance tables for all datasets are available from https://sag.cs.herts.ac.uk/?page_id=235
} 
Table 5 Performance measures for $\mathrm{KC} 4, \mathrm{KN}$ and Ivy

\begin{tabular}{|c|c|c|c|c|c|c|}
\hline \multirow[b]{2}{*}{ Classifier } & \multicolumn{2}{|l|}{$\mathrm{KC} 4$} & \multicolumn{2}{|l|}{$\mathrm{KN}$} & \multicolumn{2}{|l|}{ Ivy } \\
\hline & $\mathrm{MCC}$ & F-measure & $\mathrm{MCC}$ & F-measure & $\mathrm{MCC}$ & F-measure \\
\hline SVM & 0.567 & 0.795 & 0.400 & 0.404 & 0.141 & 0.167 \\
\hline RPart & 0.650 & 0.825 & 0.276 & 0.218 & 0.244 & 0.324 \\
\hline NB & 0.272 & 0.419 & 0.098 & 0.170 & 0.295 & 0.375 \\
\hline RF & 0.607 & 0.809 & 0.397 & 0.378 & 0.310 & 0.316 \\
\hline
\end{tabular}

defective items flipping Never $=0.717$ would indicate that $71.7 \%$ of defective items never flipped, a value of defective items flipping $<5 \%=0.746$ would indicate that $74.6 \%$ of defective items flipped less than $5 \%$ of the time. Table 7 suggests that non-defective items had a more stable prediction than defective items across all datasets. Although Table 7 shows the average numbers of prediction flipping across all datasets, this statement is valid for all of our dataset categories as shown in Table 6. This is probably because of the imbalance of data. Since there is more non-defective items to learn from, predictors could be better trained to predict them and hence flip less. Although the average numbers do not indicate much flipping between modules being predicted as defective or non-defective, these tables show datasets together, and so, the low flipping in large datasets masks the flipping that occurs in individual datasets.

Table 8 shows the label flipping variations during the 100 runs between datasets. ${ }^{12}$ For some datasets, using particular classifiers results in a high level of flipping (prediction uncertainty). For example, Table 8 shows that using Naïve Bayes on KN results in prediction uncertainty, with $73 \%$ of the predictions for known defective modules flipping at least once between being predicted defective to predicted non-defective between runs. Table 8 also shows the prediction uncertainty of using SVM on the KC4 dataset with only $26 \%$ of known defective modules being consistently predicted as defective or not defective across all cross-validation runs. Figure 2 presents violin plots showing the flipping for the four different classifiers on KC4 in more detail. ${ }^{13}$ The violin plots show the flipping that occurs for each quadrant of the confusion matrix. The $y$-axis represents the probability of a module to flip, where the central part of a 'violin' represents the 50\% chance of flipping, reducing towards no flipping at the ends of the 'violin'. The $x$-axis demonstrates the proportion of modules that flip, where a wide 'violin' indicates a high proportion of modules, and a narrow 'violin' represents a small number of modules. For example, Fig. 2 shows that SVM is particularly unstable when predicting both, defective and non-defective modules for $\mathrm{KC} 4$, compared to the other classifiers. The reason for that is the wider 'violin' body around the $50 \%$ probability of flipping. On the other hand, Naïve Bayes shows the greatest stability when predicting non-defective instances since the majority of modules are concentrated closer to the ends of the 'violin'. RPart provides relatively stable predictions for defective instances when used on the $\mathrm{KC} 4$ dataset. As a result of analysing these labelling variations between runs, we decided to label a module as having been predicted as either defective or not defective if it had been predicted as such on more than 50 runs. Using a threshold of 50 is the equivalent of choosing the label based on the balance of probability.

\footnotetext{
${ }^{12}$ Label flipping tables for all datasets are available from https://sag.cs.herts.ac.uk/?page_id=235.

${ }^{13}$ Violin plots for all datasets are available from https://sag.cs.herts.ac.uk/?page_id=235
} 
Table 6 Frequency of all items flipping across different dataset categories

\begin{tabular}{|c|c|c|c|c|c|c|c|}
\hline & \multirow[b]{2}{*}{ Classifier } & \multicolumn{3}{|c|}{ Non-defective items } & \multicolumn{3}{|c|}{ Defective items } \\
\hline & & Never & $<5 \%$ & $<10 \%$ & Never & $<5 \%$ & $<10 \%$ \\
\hline \multirow[t]{4}{*}{ NASA } & SVM & 0.983 & 0.985 & 0.991 & 0.717 & 0.746 & 0.839 \\
\hline & RPart & 0.972 & 0.972 & 0.983 & 0.626 & 0.626 & 0.736 \\
\hline & NB & 0.974 & 0.974 & 0.987 & 0.943 & 0.943 & 0.971 \\
\hline & RF & 0.988 & 0.991 & 0.993 & 0.748 & 0.807 & 0.859 \\
\hline \multirow[t]{4}{*}{ Comm } & SVM & 0.959 & 0.967 & 0.974 & 0.797 & 0.797 & 0.797 \\
\hline & RPart & 0.992 & 0.992 & 0.995 & 0.901 & 0.901 & 0.901 \\
\hline & NB & 0.805 & 0.805 & 0.879 & 0.823 & 0.823 & 0.823 \\
\hline & $\mathrm{RF}$ & 0.989 & 0.992 & 0.995 & 0.897 & 0.897 & 0.897 \\
\hline \multirow[t]{4}{*}{ OSS } & SVM & 0.904 & 0.925 & 0.942 & 0.799 & 0.799 & 0.799 \\
\hline & RPart & 0.850 & 0.850 & 0.899 & 0.570 & 0.570 & 0.570 \\
\hline & NB & 0.953 & 0.953 & 0.971 & 0.924 & 0.924 & 0.924 \\
\hline & $\mathrm{RF}$ & 0.958 & 0.970 & 0.975 & 0.809 & 0.809 & 0.809 \\
\hline
\end{tabular}

Having labelled each module as being predicted or not as defective by each of the four classifiers, we constructed set diagrams to show which defects were identified by which classifiers. Figures 3-5 show set diagrams for all dataset categories, divided in groups for NASA datasets, open source datasets, and commercial datasets, respectively. Figure 3 shows a set diagram for the 12 frequently used NASA datasets together. Each figure is divided into the four quadrants of a confusion matrix. The performance of each individual classifier is shown in terms of the numbers of predictions falling into each quadrant. Figures 3-5 show similarity and variation in the actual modules predicted as either defective or not defective by each classifier. Figure 3 shows that 96 out of 1568 defective modules are correctly predicted as defective by all four classifiers (only 6.1\%). Very many more modules are correctly identified as defective by individual classifiers. For example, Naïve Bayes is the only classifier to correctly find $280(17.9 \%)$ defective modules and SVM is the only classifier to correctly locate $125(8.0 \%)$ defective modules (though such predictive performance must always be weighed against false positive predictions). Our results suggest that using only a Random Forest classifier would fail to predict many (526 (34\%)) defective modules. Observing Figs. 4 and 5 we came to similar conclusions. In the case of the open source datasets, 55 out of $283(19.4 \%)$ unique defects were identified by either Naïve Bayes or SVM. Many more unique defects were found by individual classifiers in the commercial datasets, precisely 357 out of 1027 (34.8\%).

Table 7 Frequency of all item flipping in all datasets

\begin{tabular}{lllllllll}
\hline & \multicolumn{3}{l}{ Non-defective items } & & \multicolumn{3}{l}{ Defective items } \\
\cline { 2 - 4 } \cline { 7 - 8 } Classifier & Never & $<5 \%$ & $<10 \%$ & & Never & $<5 \%$ & $<10 \%$ \\
\hline SVM & 0.949 & 0.959 & 0.969 & & 0.771 & 0.781 & 0.812 \\
RPart & 0.938 & 0.938 & 0.959 & & 0.699 & 0.699 & 0.736 \\
NB & 0.911 & 0.911 & 0.945 & & 0.897 & 0.897 & 0.906 \\
RF & 0.978 & 0.984 & 0.988 & & 0.818 & 0.838 & 0.855 \\
\hline
\end{tabular}


Table 8 Frequency of flipping for three different datasets

\begin{tabular}{|c|c|c|c|c|c|c|c|}
\hline & \multirow[b]{2}{*}{ Classifier } & \multicolumn{3}{|c|}{ Non-defective items } & \multicolumn{3}{|c|}{ Defective items } \\
\hline & & Never & $<5 \%$ & $<10 \%$ & Never & $<5 \%$ & $<10 \%$ \\
\hline \multirow[t]{4}{*}{$\mathrm{KC} 4$} & SVM & 0.719 & 0.734 & 0.828 & 0.262 & 0.311 & 0.443 \\
\hline & RPart & 0.984 & 0.984 & 1.000 & 0.902 & 0.902 & 0.984 \\
\hline & NB & 0.938 & 0.938 & 0.984 & 0.885 & 0.885 & 0.934 \\
\hline & $\mathrm{RF}$ & 0.906 & 0.938 & 0.953 & 0.803 & 0.820 & 0.918 \\
\hline \multirow[t]{4}{*}{$\mathrm{KN}$} & SVM & 0.955 & 0.964 & 0.971 & 0.786 & 0.817 & 0.854 \\
\hline & RPart & 0.993 & 0.993 & 0.997 & 0.888 & 0.888 & 0.929 \\
\hline & NB & 0.491 & 0.491 & 0.675 & 0.571 & 0.571 & 0.730 \\
\hline & $\mathrm{RF}$ & 0.988 & 0.991 & 0.994 & 0.919 & 0.922 & 0.957 \\
\hline \multirow[t]{4}{*}{ Ivy } & SVM & 0.913 & 0.949 & 0.962 & 0.850 & 0.850 & 0.875 \\
\hline & RPart & 0.837 & 0.837 & 0.881 & 0.625 & 0.625 & 0.700 \\
\hline & NB & 0.933 & 0.933 & 0.952 & 0.950 & 0.950 & 1.000 \\
\hline & $\mathrm{RF}$ & 0.955 & 0.974 & 0.981 & 0.900 & 0.925 & 0.950 \\
\hline
\end{tabular}

There is much more agreement between classifiers about non-defective modules. In the true negative quadrant, Fig. 3 shows that all four classifiers agree on 35364 (93.1\%) out of 37987 true negative NASA modules. Though again, individual non-defective modules are located by specific classifiers. For example, Fig. 3 shows that SVM correctly predicts 100 non-defective NASA modules that no other classifier predicts. The pattern of module predictions across the classifiers varies slightly between the datasets. Figures 6, 7 and 8 show set diagrams for individual datasets, KC4, KN and Ivy. Particularly, Fig. 6 shows a set diagram for the $\mathrm{KC} 4$ dataset. ${ }^{14} \mathrm{KC} 4$ is an interesting dataset. It is unusually balanced between defective and non-defective modules (64 v 61). It is also a small dataset (only 125 modules). Figure 6 shows that for KC4 Naïve Bayes behaves differently compared to how it behaves for the other datasets. In particular for KC4 Naïve Bayes is much less optimistic (i.e. it predicts only 17 out of 125 modules as being defective) in its predictions than it is for the other datasets. RPart was more conservative when predicting defective items than non-defective ones. For example, in the $\mathrm{KN}$ dataset, RPart is the only classifier to find 17 $(5.3 \%)$ unique non-defective items as shown on Fig. 8.

\section{Discussion}

Our results suggest that there is uncertainty in the predictions made by classifiers. We have demonstrated that there is a surprising level of prediction flipping between cross-validation runs by classifiers. This level of uncertainty is not usually observable as studies normally only publish average final prediction figures. Few studies concern themselves with the results of individual cross-validation runs. Elish and Elish (2008) is a notable exception to this, where the mean and the standard deviation of the performance values across all runs are reported. Few studies run experiments 100 times. More commonly, experiments are run only 10 times (e.g. Lessmann et al. (2008); Menzies et al. (2007)). This means that the level of

\footnotetext{
${ }^{14}$ Set diagrams for all datasets can be found at https://sag.cs.herts.ac.uk/?page_id=235
} 


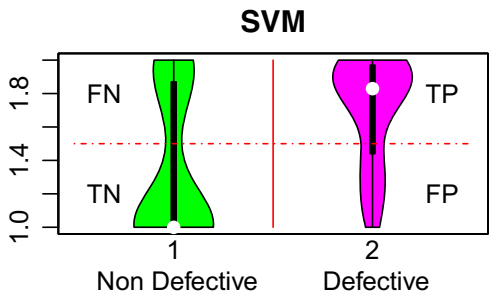

NaiveBayes

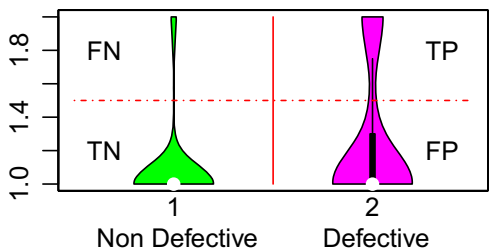

RPart

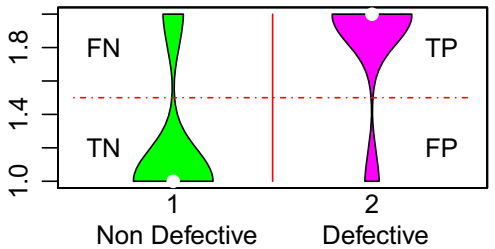

RandomForest

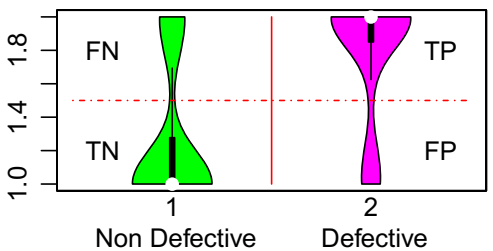

Fig. 2 Violin plot of frequency of flipping for KC4 dataset

False Negativies $=1465$

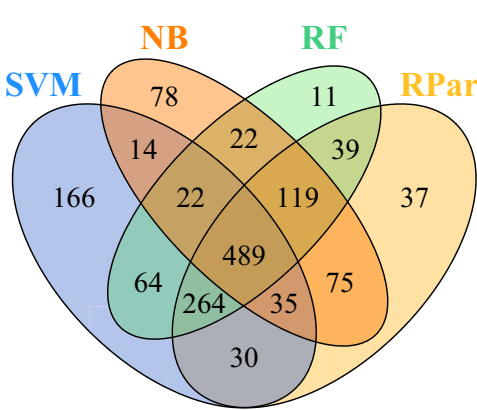

True Negativies $=37953$

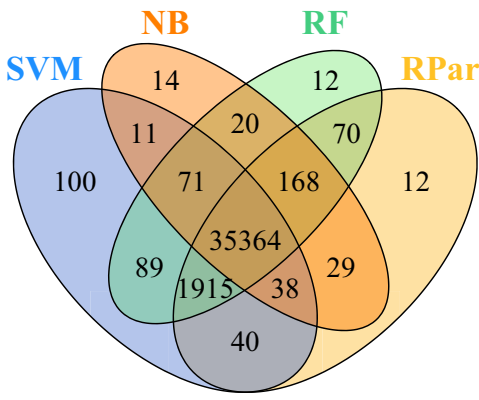

True Positives $=1064$

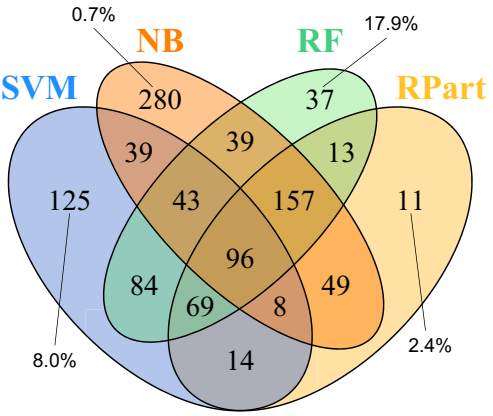

False Positives $=2553$

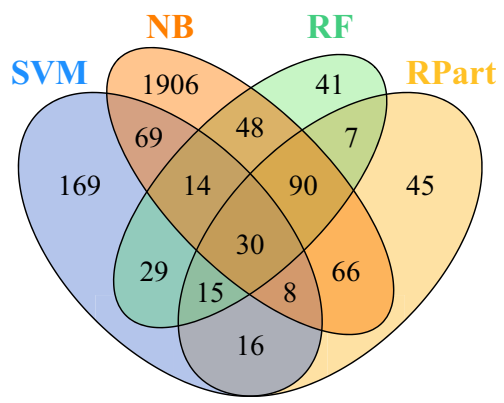

Fig. 3 Sensitivity analysis for all NASA datasets using different classifiers. $n=37987 ; p=1568$ 


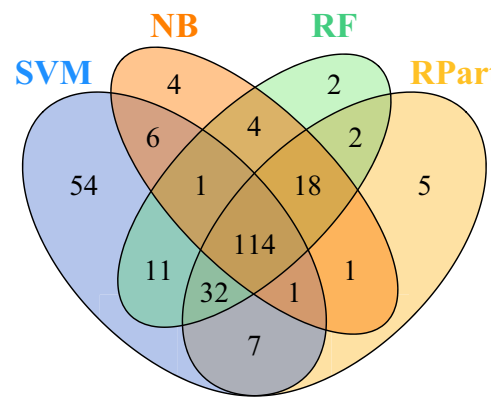

True Negativies $=1646$

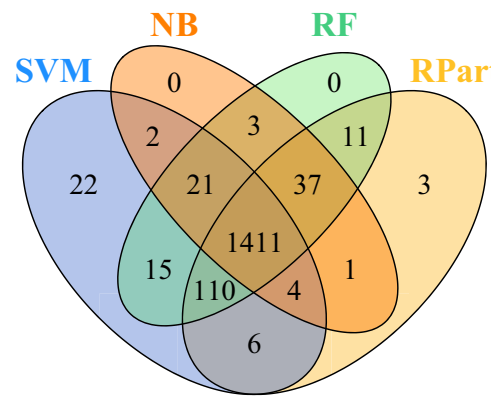

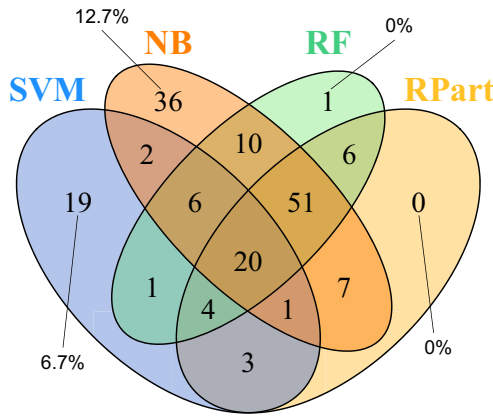

False Positives $=237$

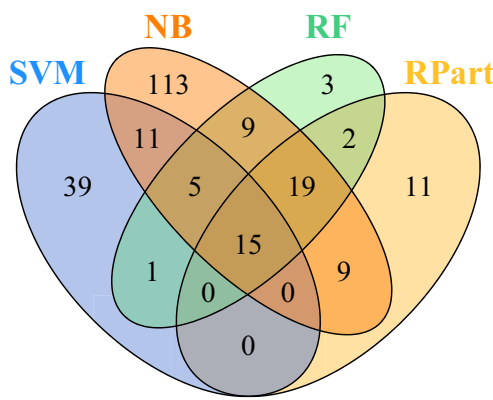

Fig. 4 Sensitivity analysis for all open source datasets using different classifiers. $n=1663 ; \mathrm{p}=283$

prediction flipping between runs is likely to be artificially reduced. We suspect that prediction flipping by a classifier for a dataset is caused by the random generation of the folds. The items making up the individual folds determine the composition of the training data and the model that is built. The larger the dataset, the less prediction flipping occurs. This is likely to be because larger datasets may have training data that is more consistent with the entire dataset. Some classifiers are more sensitive to the composition of the training set than other classifiers. SVM is particularly sensitive for KC4 where $26 \%$ of non-defective items flip at least once and $44 \%$ of defective items flip. Although SVM performs well (MCC $=0.567)$, the items it predicts as being defective are not consistent across different cross-validation runs. A similar situation is observed with Ant, where the level of flipping for Rpart is 63\% while maintaining a reasonable performance $(\mathrm{MCC}=0.398)$. However, the reasons for such prediction uncertainty remain unknown and investigating the cause of this uncertainty is beyond the scope of this paper. Future research is also needed to use our results on flipping to identify the threshold at which overall defective or not defective predictions should be determined.

The level of uncertainty among classifiers may be valuable for practitioners in different domains of defect predictions. For instance, where stability of prediction plays a significant role, our results suggest that on average, Naïve Bayes would be the most suitable selection. On the other hand, learners such as RPart may be avoided in applications where higher prediction consistency is needed. The reasons for this prediction inconsistency are 
False Negatives $=1001$

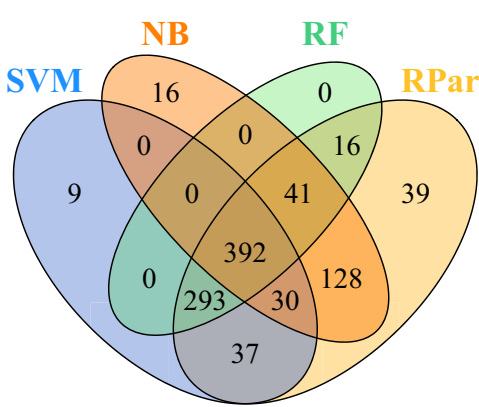

True Negatives $=17337$

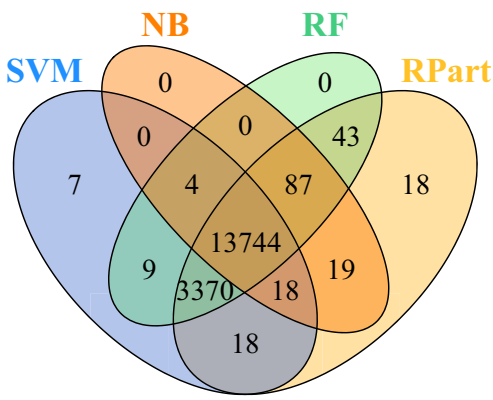

True Positives $=628$

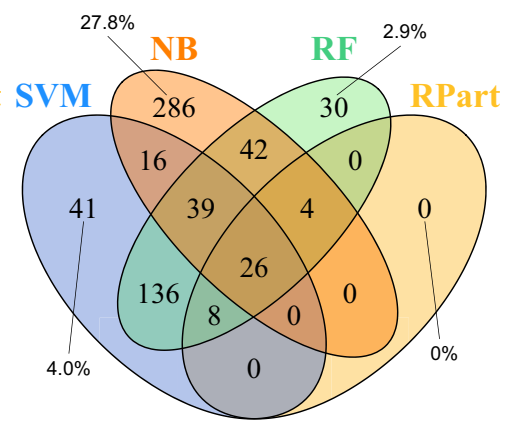

False Positivies $=3456$

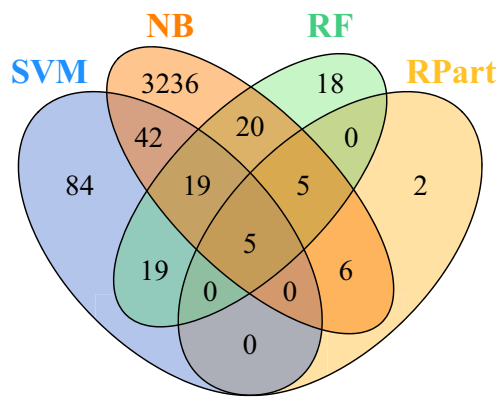

Fig. 5 Sensitivity analysis for all commercial datasets using different classifiers. $n=17344 ; \mathrm{p}=1027$

yet to be established. More classifiers with different properties should also be investigated to establish the extent of uncertainty in predictions.

Other large-scale studies comparing the performance of defect prediction models show that there is no significant difference between classifiers (Arisholm et al. 2010; Lessmann et al. 2008). Our overall MCC values for the four classifiers we investigate also suggest performance similarity. Our results show that specific classifiers are sensitive to dataset and that classifier performance varies according to dataset. For example, our SVM model performs poorly on Ivy but performs much better on $\mathrm{KC} 4$. Other studies have also reported sensitivity to dataset (e.g. Lessmann et al. (2008)).

Similarly to Panichella et al. (2014), our results also suggest that overall performance figures hide a variety of differences in the defects that each classifier predicts. While overall performance figures between classifiers are similar, very different subsets of defects are actually predicted by different classifiers. So, it would be wrong to conclude that, given overall performance values for classifiers are similar, it does not matter which classifier is used. Very different defects are predicted by different classifiers. This is probably not surprising given that the four classifiers we investigate approach the prediction task using very different techniques. From each category of system in our analysis, we observe a considerable number of defects predicted by a single classifier. Overall, the NASA category contains $43 \%$, Comm $57 \%$ and OSS $34 \%$ of unique defects predicted by only one classifier. Future work is needed to investigate whether there is any similarity in the characteristics of 


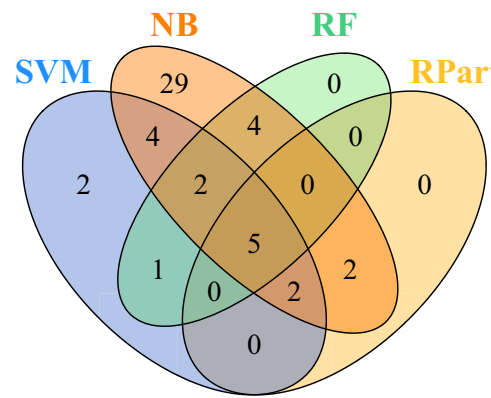

True Negativies $=61$

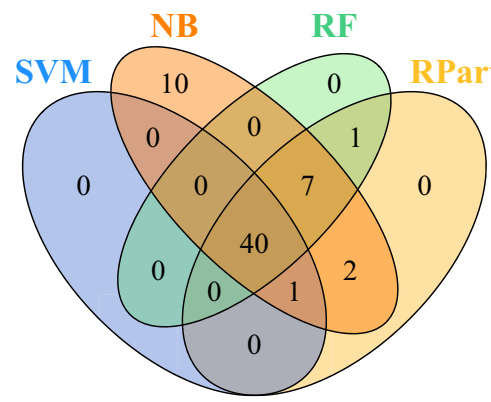

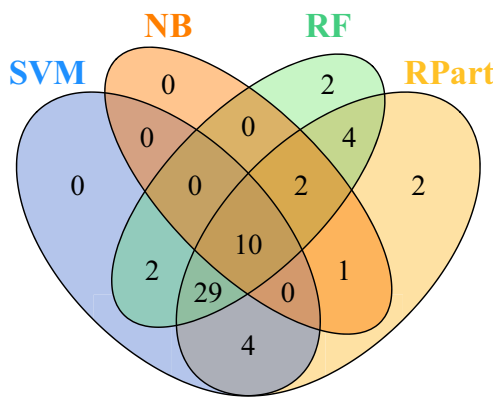

False Positivies $=24$

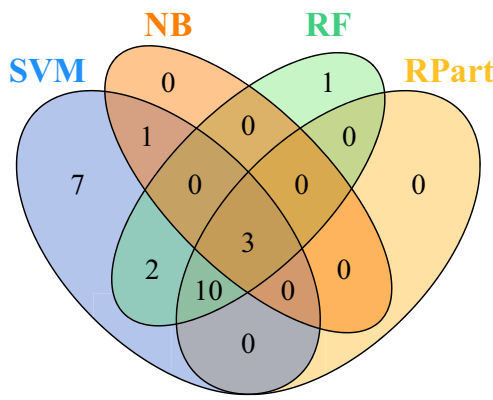

Fig. 6 Sensitivity analysis for $\mathrm{KC} 4$ using different classifiers. $n=64 ; p=61$

the set of defects that each classifier predicts. Currently, it is not known whether particular classifiers specialise in predicting particular types of defect.

Our results strongly suggest the use of classifier ensembles. It is likely that a collection of heterogeneous classifiers offer the best opportunity to predict defects. Future work is needed to extend our investigation and identify which set of classifiers perform best in terms of prediction performance and consistency. This future work also needs to identify whether a global ensemble could be identified or whether effective ensembles remain local to the dataset. Our results also suggest that ensembles should not use the popular majority voting approach to deciding on predictions. Using this decision-making approach will miss the unique subsets of defects that individual classifiers predict. Such understanding has previously not been obvious since only average overall performance figures for different classifiers have been reported. Our results now support Kim et al. (2011)'s recommendations on the use of classifier ensembles, and we, in addition, provide better understanding about ensemble design. One way forward in building future prediction models could be stacking ensembles. The stacking approach does not base its predictions on voting, but rather uses an additional classifier to make the final prediction. Our recent study has shown that stacking ensembles provide significantly better prediction performance compared to many other classifiers (Petrić et al. 2016a). However, a substantial amount of future work is needed to establish a decision- making approach for ensembles that will fully exploit our findings. Our results further indicate the possible reasons for high false alarms previously attributed to 


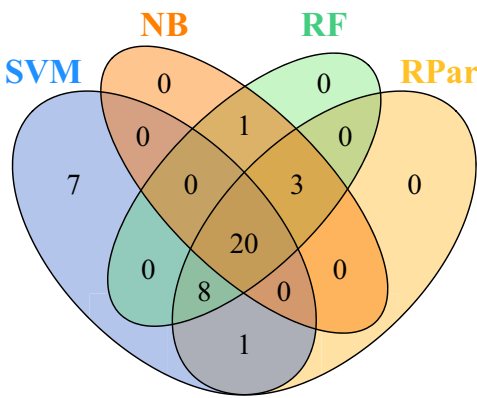

True Negativies $=312$
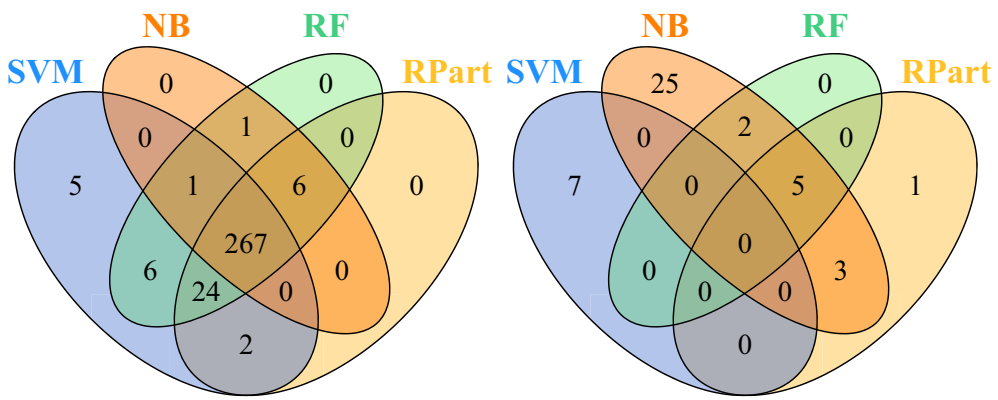

Fig. 7 Ivy sensitivity analysis using different classifiers. $n=312 ; p=40$

ensembles. As many true defects are individually predicted by single classifiers, ensembles based on majority voting approach would certainly misclassify such defects.

\section{Threats to validity}

Although we implemented what could be regarded as current best practice in classifierbased model building, there are many different ways in which a classifier may be built. There are also many different ways in which the data used can be pre-processed. All of these factors are likely to impact on predictive performance. As Lessmann et al. (2008) say classification is only a single step within a multistage data mining process (Fayyad et al. 1996). Especially, data preprocessing or engineering activities such as the removal of non-informative features or the discretisation of continuous attributes may improve the performance of some classifiers (see, e.g., Dougherty et al. (1995) and Hall and Holmes (2003)). Such techniques have an undisputed value. Despite the likely advantages of implementing these many additional techniques, as Lessmann et al. we implemented only a basic set of these techniques. Our reason for this decision was the same as Lessmann et al....computationally infeasible when considering a large number of classifiers at the same time. The experiments we report here each took several days of processing time. We did implement a set of techniques that are commonly used in defect prediction of which there is evidence they improve predictive performance. We went further in some of the techniques 


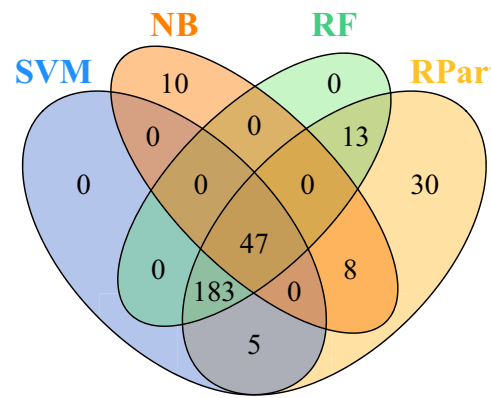

True Negativies $=3985$

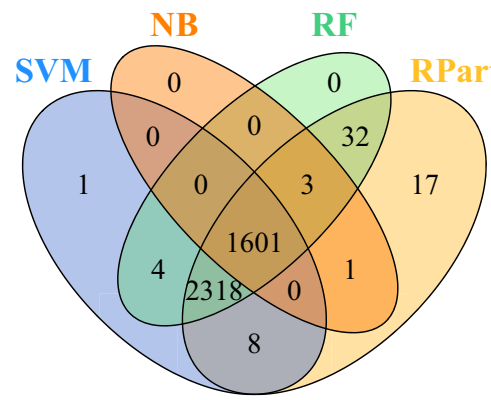

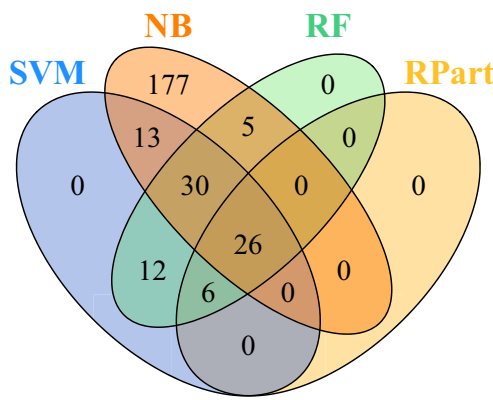

False Positivies $=2274$

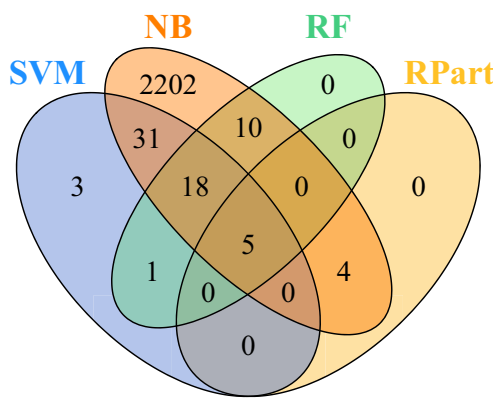

Fig. 8 KN sensitivity analysis using different classifiers. $n=3992 ; p=322$

we implemented, e.g. running our experiments 100 times rather than the 10 times that studies normally do. However, we did not implement a technique to address data imbalance (e.g. SMOTE). This was because data imbalance does not affect all classifiers equally. We implemented only partial feature reduction. The impact of the model building and data pre-processing approaches we used are not likely to significantly affect the results we report. This could be due to the ceiling effect reported in 2008, which states that prediction modelling solely based on model building and data pre-processing cannot break through the performance ceiling (Menzies et al. 2008). In addition, the range of steps we applied in our experiments while building prediction models are comparable to current defect prediction studies (e.g. repeated experiments, the use of cross validation, etc.).

Our studies are also limited in that we only investigated four classifiers. It may be that there is less variation in the defect subsets detected by classifiers that we did not investigate. We believe this to be unlikely, as the four classifiers we chose are representative of discrete groupings of classifiers in terms of the prediction approaches used. However, future work will have to determine whether additional classifiers behave as we report these four classifiers to. We also used a limited number of datasets in our study. Again, it is possible that other datasets behave differently. We believe this will not be the case, as the 18 datasets we investigated were wide ranging in their features and produced a variety of results in our investigation.

Our analysis is also limited by only measuring predictive performance using f-measure and MCC metrics. Such metrics are implicitly based on the cut-off points used by the 
classifiers themselves to decide whether a software component is defective or not. All software components having a defective probability above a certain cut-off point (in general, it is equal to 0.5) are labelled as 'defective', or as 'non-defective' otherwise. For example, Random Forest not only provides a binary classification of datapoints but also provides the probabilities for each component belonging to 'defective' or 'non-defective' categories. D'Ambros et al. (2012) investigated the effect of different cut-off points on the performances of classification algorithms in the context of defect prediction and proposed other performance metrics that are independent from the specific (and also implicit) cut-off points used by different classifiers. Future work includes consideration of the different cut-off points to the individual performances of the four classifiers used in this paper.

\section{Conclusion}

We report a surprising amount of prediction variation within experimental runs. We repeated our cross-validation runs 100 times. Between these runs, we found a great deal of inconsistency in whether a module was predicted as defective or not by the same model. This finding has important implications for defect prediction as many studies only repeat experiments 10 times. This means that the reliability of some previous results may be compromised. In addition, the prediction flipping that we report has implications for practitioners. Although practitioners may be happy with the overall predictive performance of a given model, they may not be so happy that the model predicts different modules as defective depending on the training of the model. Our analysis shows that the classifier's inconsistency occurs in a variety of different software domains, including open source and commercial projects.

Performance measures can make it seem that defect prediction models are performing similarly. However, even where similar performance figures are produced, different defects are identified by different classifiers. This has important implications for defect prediction. First, assessing predictive performance using conventional measures such as f-measure, precision or recall gives only a basic picture of the performance of models. Second, models built using only one classifier are not likely to comprehensively detect defects. Ensembles of classifiers need to be used. Third, current approaches to ensembles need to be re-considered. In particular, the popular 'majority' voting decision approach used by ensembles will miss the sizeable subsets of defects that single classifiers correctly predict. Ensemble decisionmaking strategies need to be enhanced to account for the success of individual classifiers in finding specific sets of defects. Our results support the use of classifier ensembles not based on majority voting.

The feature selection techniques for each classifier could also be explored in the future. Since different classifiers find different subsets of defects, it is reasonable to explore whether some particular features better suit specific classifiers. Perhaps some classifiers work better when combined with specific subsets of features.

We suggest new ways of building enhanced defect prediction models and opportunities for effectively evaluating the performance of those models in within-project studies. These opportunities could provide future researchers with the tools with which to break through the performance ceiling currently being experienced in defect prediction.

Acknowledgments This work was partly funded by a grant from the UK's Engineering and Physical Sciences Research Council under grant number: EP/L011751/1. 
Open Access This article is distributed under the terms of the Creative Commons Attribution 4.0 International License (http://creativecommons.org/licenses/by/4.0/), which permits unrestricted use, distribution, and reproduction in any medium, provided you give appropriate credit to the original author(s) and the source, provide a link to the Creative Commons license, and indicate if changes were made.

\section{References}

Arisholm, E., \& Briand, L.C. (2007). Fuglerud M Data mining techniques for building fault-proneness models in telecom java software. In Software Reliability, 2007. ISSRE '07. The 18th IEEE International Symposium on, pp 215-224.

Arisholm, E., Briand, L.C., \& Johannessen, E.B. (2010). A systematic and comprehensive investigation of methods to build and evaluate fault prediction models. Journal of Systems and Software, 83(1), 2 17.

Bell, R., Ostrand, T., \& Weyuker, E. (2006). Looking for bugs in all the right places. In Proceedings of the 2006 international symposium on Software testing and analysis, ACM, pp 61-72.

Bibi, S., Tsoumakas, G., Stamelos, I., \& Vlahvas, I. (2006). Software defect prediction using regression via classification. In IEEE international conference on computer systems and applications.

Bird, C., Bachmann, A., Aune, E., Duffy, J., Bernstein, A., Filkov, V., \& Devanbu, P. (2009a). Fair and balanced?: bias in bug-fix datasets.In Proceedings of the the 7th joint meeting of the European software engineering conference and the ACM SIGSOFT symposium on The foundations of software engineering, ACM, New York, NY, USA, ESEC/FSE '09, pp 121-130.

Bird, C., Nagappan, N., Gall, H., Murphy, B., \& Devanbu, P. (2009b). Putting it all together: Using socio-technical networks to predict failures. In 20th International Symposium on Software Reliability Engineering, IEEE, pp 109-119.

Boetticher, G. (2006). Advanced machine learner applications in software engineering, Idea Group Publishing, Hershey, PA, USA, chap Improving credibility of machine learner models in software engineering.

Bowes, D., Hall, T., \& Gray, D. (2013). DConfusion: a technique to allow cross study performance evaluation of fault prediction studies. Automated Software Engineering, 1-27. doi:10.1007/s10515-013-0129-8.

Bowes, D., Hall, T., \& Petrić, J. (2015). Different classifiers find different defects although with different level of consistency. In Proceedings of the 11th International Conference on Predictive Models and Data Analytics in Software Engineering, PROMISE '15, pp 3:1-3:10. doi:10.1145/2810146.2810149.

Briand, L., Melo, W., \& Wust, J. (2002). Assessing the applicability of fault-proneness models across objectoriented software projects. IEEE Transactions on Software Engineering, 28(7), 706-720.

Catal, C., \& Diri, B. (2009). A systematic review of software fault prediction studies. Expert Systems with Applications, 36(4), 7346-7354.

Chawla, N.V., Japkowicz, N., \& Kotcz, A. (2004). Editorial: special issue on learning from imbalanced data sets. SIGKDD Explorations, 6(1), 1-6.

Chen, H., \& Yao, X. (2009). Regularized negative correlation learning for neural network ensembles. IEEE Transactions on Neural Networks, 20(12), 1962-1979.

Chen, W., Wang, Y., Cao, G., Chen, G., \& Gu, Q. (2014). A random forest model based classification scheme for neonatal amplitude-integrated eeg. Biomedical engineering online, 13(2), 1.

D’Ambros, M., Lanza, M., \& Robbes, R. (2009). On the relationship between change coupling and software defects. In 16th working conference on reverse engineering, 2009. WCRE '09., pp 135-144.

D’Ambros, M., Lanza, M., \& Robbes, R. (2012). Evaluating defect prediction approaches: a benchmark and an extensive comparison. Empirical Software Engineering, 17(4), 531-577. doi:10.1007/s10664-0119173-9.

Dougherty, J., Kohavi, R., \& Sahami, M. (1995). Supervised and unsupervised discretization of continuous features. In ICML, pp 194-202.

Elish, K., \& Elish, M. (2008). Predicting defect-prone software modules using support vector machines. Journal of Systems and Software, 81(5), 649-660.

Fayyad, U., Piatetsky-Shapiro, G., \& Smyth, P. (1996). From data mining to knowledge discovery in databases. AI magazine, 17(3), 37.

Fenton, N., \& Neil, M. (1999). A critique of software defect prediction models. IEEE Transactions on Software Engineering, 25(5), 675-689.

Gao, K., Khoshgoftaar, T.M., \& Napolitano, A. (2015). Combining feature subset selection and data sampling for coping with highly imbalanced software data. In Proc. of 27th International Conf. on Software Engineering and Knowledge Engineering, Pittsburgh. 
Gray, D. (2013). Software defect prediction using static code metrics : Formulating a methodology. University of Hertfordshire: PhD thesis, Computer Science.

Gray, D., Bowes, D., Davey, N., Sun, Y., \& Christianson, B. (2011). The misuse of the NASA metrics data program data sets for automated software defect prediction. In EASE 2011, IET, Durham, UK.

Gray, D., Bowes, D., Davey, N., Sun, Y., \& Christianson, B. (2012). Reflections on the NASA MDP data sets. IET Software, 6(6), 549-558.

Hall, M.A., \& Holmes, G. (2003). Benchmarking attribute selection techniques for discrete class data mining. IEEE Transactions on Knowledge and Data Engineering, 15(6), 1437-1447.

Hall, T., Beecham, S., Bowes, D., Gray, D., \& Counsell, S. (2012). A systematic literature review on fault prediction performance in software engineering. IEEE Transactions on Software Engineering, 38(6), 1276-1304.

Jiang, Y., Lin, J., Cukic, B., \& Menzies, T. (2009). Variance analysis in software fault prediction models. In SSRE 2009, 20th International Symposium on Software Reliability Engineering, IEEE Computer Society, Mysuru, Karnataka, India, 16-19 November 2009, pp 99-108.

Jureczko, M., \& Madeyski, L. (2010). Towards identifying software project clusters with regard to defect prediction. In Proceedings of the 6th International Conference on Predictive Models in Software Engineering, ACM, New York, NY, USA, PROMISE '10, pp 9:1-9:10. doi:10.1145/1868328.1868342.

Kamei, Y., \& Shihab, E. (2016). Defect prediction: Accomplishments and future challenges. In 2016 IEEE $23 r d$ international conference on software analysis, evolution, and reengineering (SANER), vol 5, pp 33-45. doi:10.1109/SANER.2016.56.

Khoshgoftaar, T., Yuan, X., Allen, E., Jones, W., \& Hudepohl, J. (2002). Uncertain classification of faultprone software modules. Empirical Software Engineering, 7(4), 297-318.

Khoshgoftaar, T.M., Gao, K., \& Seliya, N. (2010). Attribute selection and imbalanced data: Problems in software defect prediction. In 2010 22nd IEEE international conference on tools with artificial intelligence (ICTAI), vol 1, pp 137-144.

Kim, S., Zhang, H., Wu, R., \& Gong, L. (2011). Dealing with noise in defect prediction. In Proceedings of the 33rd International Conference on Software Engineering, ACM, New York, NY, USA, ICSE '11, pp $481-490$.

Kutlubay, O., Turhan, B., \& Bener, A. (2007). A two-step model for defect density estimation. In 33rd EUROMICRO Conference on Software Engineering and Advanced Applications, 2007., pp 322332.

Laradji, I.H., Alshayeb, M., \& Ghouti, L. (2015). Software defect prediction using ensemble learning on selected features. Information and Software Technology, 58, 388-402. doi:10.1016/j.infsof.2014.07.005. http://www.sciencedirect.com/science/article/pii/S0950584914001591.

Lessmann, S., Baesens, B., Mues, C., \& Pietsch, S. (2008). Benchmarking classification models for software defect prediction: A proposed framework and novel findings. IEEE Transactions on Software Engineering, 34(4), 485-496.

Liebchen, G., \& Shepperd, M. (2008). Data sets and data quality in software engineering. In Proceedings of the 4th international workshop on Predictor models in software engineering, ACM, pp 3944.

Madeyski, L., \& Jureczko, M. (2015). Which process metrics can significantly improve defect prediction models? An empirical study. Software Quality Journal, 23(3), 393-422. doi:10.1007/s11219-014-9241-7.

Malhotra, R. (2015). A systematic review of machine learning techniques for software fault prediction. Appl Soft Comput, 27(C), 504-518. doi:10.1016/j.asoc.2014.11.023.

Mende, T. (2011). On the evaluation of defect prediction models. In The 15th CREST Open Workshop.

Mende, T., \& Koschke, R. (2010). Effort-aware defect prediction models. In Software Maintenance and Reengineering (CSMR), 2010 14th European Conference on, pp 107-116.

Menzies, T., Greenwald, J., \& Frank, A. (2007). Data mining static code attributes to learn defect predictors. IEEE Transactions on Software Engineering, 33(1), 2-13.

Menzies, T., Turhan, B., Bener, A., Gay, G., Cukic, B., \& Jiang, Y. (2008). Implications of ceiling effects in defect predictors. In Proceedings of the 4th international workshop on Predictor models in software engineering, pp 47-54.

Menzies, T., Caglayan, B., He, Z., Kocaguneli, E., Krall, J., Peters, F., \& Turhan, B. (2012). The promise repository of empirical software engineering data. http://promisedata.googlecode.com.

Minku, L.L., \& Yao, X. (2012). Ensembles and locality: Insight on improving software effort estimation. Information and Software Technology.

Minku, L.L., \& Yao, X. (2013). Software effort estimation as a multi-objective learning problem. ACM Transactions on Software Engineering and Methodology. to appear.

Misirlı, A.T., Bener, A.B., \& Turhan, B. (2011). An industrial case study of classifier ensembles for locating software defects. Software Quality Journal, 19(3), 515-536. 
Mizuno, O., \& Kikuno, T. (2007). Training on errors experiment to detect fault-prone software modules by spam filter. In Proceedings of the the 6th joint meeting of the European software engineering conference and the ACM SIGSOFT symposium on The foundations of software engineering, ACM, New York, NY, USA, ESEC-FSE '07, pp 405-414.

Mizuno, O., Ikami, S., Nakaichi, S., \& Kikuno, T. (2007). Spam filter based approach for finding faultprone software modules. In Mining Software Repositories, 2007. ICSE Workshops MSR '07. Fourth International Workshop on, $p 4$.

Myrtveit, I., Stensrud, E., \& Shepperd, M. (2005). Reliability and validity in comparative studies of software prediction models. IEEE Transactions on Software Engineering, 380-391.

Nagappan, N., Zeller, A., Zimmermann, T., Herzig, K., \& Murphy, B. (2010). Change bursts as defect predictors. In Software Reliability Engineering, 2010 IEEE 21 st International Symposium on, pp 309-318.

Ostrand, T., Weyuker, E., \& Bell, R. (2010). Programmer-based fault prediction. In Proceedings of the 6th International Conference on Predictive Models in Software Engineering, ACM, pp 1-10.

Panichella, A., Oliveto, R., \& De Lucia, A. (2014). Cross-project defect prediction models: L'union fait la force. In 2014 Software Evolution Week - IEEE Conference on Software Maintenance, Reengineering and Reverse Engineering (CSMR-WCRE), pp 164-173. doi:10.1109/CSMR-WCRE.2014.6747166.

Petrić, J., Bowes, D., Hall, T., Christianson, B., \& Baddoo, N. (2016). Building an ensemble for software defect prediction based on diversity selection. In The 10th International Symposium on Empirical Software Engineering and Measurement, ESEM'16, p 10.

Petrić, J., Bowes, D., Hall, T., Christianson, B., \& Baddoo, N. (2016). The jinx on the NASA software defect data sets. In Proceedings of the 20th International Conference on Evaluation and Assessment in Software Engineering, ACM, New York, NY, USA, EASE '16, pp 13:1-13:5. doi:10.1145/2915970.2916007.

Rodriguez, D., Herraiz, I., Harrison, R., Dolado, J., \& Riquelme, J.C. (2014). Preliminary comparison of techniques for dealing with imbalance in software defect prediction. In Proceedings of the 18th International Conference on Evaluation and Assessment in Software Engineering, ACM, New York, NY, USA, EASE' 14, pp 43:1-43:10. doi:10.1145/2601248.2601294.

Seiffert, C., Khoshgoftaar, T.M., \& Hulse, J.V. (2009). Improving software-quality predictions with data sampling and boosting. IEEE Transactions on Systems, Man, and Cybernetics Part A, 39(6), 1283-1294.

Shepperd, M., \& Kadoda, G. (2001). Comparing software prediction techniques using simulation. IEEE Transactions on Software Engineering, 27(11), 1014-1022.

Shepperd, M., Song, Q., Sun, Z., \& Mair, C. (2013). Data quality: some comments on the NASA software defect datasets. IEEE Transactions on Software Engineering, 39(9), 1208-1215. doi:10.1109/TSE.2013. 11.

Shepperd, M., Bowes, D., \& Hall, T. (2014). Researcher bias: The use of machine learning in software defect prediction. IEEE Transactions on Software Engineering, 40(6), 603-616. doi:10.1109/TSE.2014.232 358.

Shin, Y., Bell, R.M., Ostrand, T.J., \& Weyuker, E.J. (2009). Does calling structure information improve the accuracy of fault prediction? In Godfrey, M.W., \& Whitehead, J. (Eds.) Proceedings of the 6th International Working Conference on Mining Software Repositories, IEEE, pp 61-70.

Shivaji, S., Whitehead, E.J., Akella, R., \& Sunghun, K. (2009). Reducing features to improve bug prediction. In Automated Software Engineering, 2009. ASE '09. 24th IEEE/ACM International Conference on, pp $600-604$.

Soares, C., Brazdil, P.B., \& Kuba, P. (2004). A meta-learning method to select the kernel width in support vector regression. Machine learning, 54(3), 195-209.

Sun, Z., Song, Q., \& Zhu, X. (2012). Using coding-based ensemble learning to improve software defect prediction. IEEE Transactions on Systems, Man, and Cybernetics, Part C: Applications and Reviews, 42(6), 1806-1817. doi:10.1109/TSMCC.2012.2226152.

Turhan, B., Menzies, T., Bener, A.B., \& Di Stefano, J. (2009). On the relative value of crosscompany and within-company data for defect prediction. Empirical Softw Engg, 14(5), 540-578. doi:10.1007/s 10664-008-9103-7.

Visa, S., \& Ralescu, A. (2004). Fuzzy classifiers for imbalanced, complex classes of varying size. In Information Processing and Management of Uncertainty in Knowledge-Based Systems, pp 393-400.

Wahono, R. (2015). A systematic literature review of software defect prediction: research trends, datasets, methods and frameworks. Journal of Software Engineering, 1(1). http://journal.ilmukomputer.org/index. $\mathrm{php} / \mathrm{jse} / \mathrm{article} / \mathrm{view} / 47$.

Witten, I. (2005). Frank E. Data mining: practical machine learning tools and techniques: Morgan Kaufmann.

Wolpert, D.H. (1992). Stacked generalization. Neural Networks, 5(2), 241-259. doi:10.1016/S0893-6080 (05)80023-1.

Zhang, H. (2009). An investigation of the relationships between lines of code and defects. In Software Maintenance, 2009. ICSM 2009. IEEE International Conference on, pp 274-283. 
Zhou, Y., Xu, B., \& Leung, H. (2010). On the ability of complexity metrics to predict fault-prone classes in object-oriented systems. Journal of Systems and Software, 83(4), 660-674.

Zimmermann, T., Nagappan, N., Gall, H., Giger, E., \& Murphy, B. (2009). Cross-project defect prediction: a large scale experiment on data vs. domain vs. process. In Proceedings of the the 7th joint meeting of the European software engineering conference and the ACM SIGSOFT symposium on The foundations of software engineering, ACM, New York, NY, USA, ESEC/FSE '09, pp 91-100.

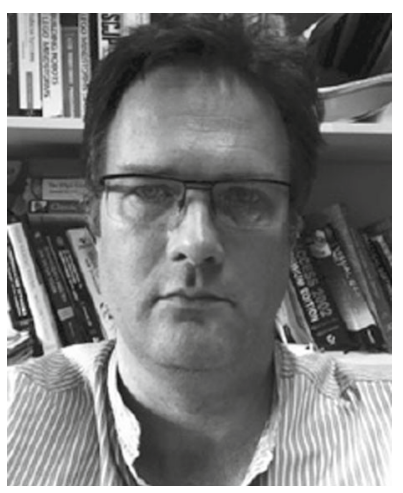

David Bowes received the $\mathrm{PhD}$ degree in defect prediction and is currently a Senior Lecturer in software engineering at the University of Hertfordshire. He has published mainly in the area of static code metrics and defect prediction. His research interests include the reliability of empirical studies.

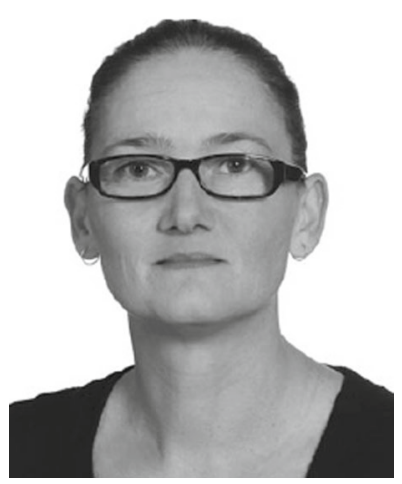

Tracy Hall received the $\mathrm{PhD}$ degree in the implementation of software metrics from City University, London. She is currently a Professor in software engineering at Brunel University London. Her expertise is in empirical software engineering and her current research activities are focused on software fault prediction. She has published more than 100 international peer-reviewed papers. 


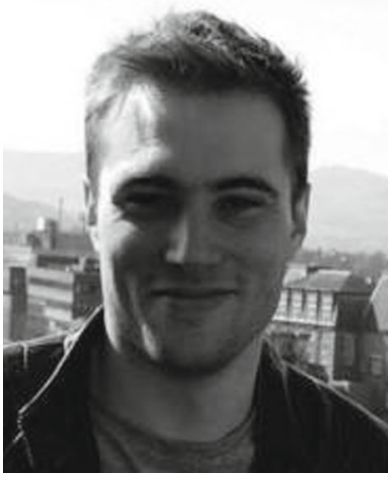

Jean Petrić received the MSc degree in Computer Science from the University of Rijeka, Croatia. He has been working towards his $\mathrm{PhD}$ at the University of Hertfordshire, and is currently a research fellow at Brunel University London. His research interest is in software defect prediction. 